\title{
外来植物入侵对土壤氮有效性的影响
}

\author{
许 浩 胡朝臣许士麒 孙新超 刘学炎”
}

天津大学表层地球系统科学研究院, 天津 300072

\begin{abstract}
摘 要 外来植物入侵对土壤氮循环和氮有效性的影响是入侵成功或进一步加剧的重要原因。通过对比相同研究地点入侵区 域和无入侵区域的土壤原位氮状态差异，探讨了外来植物入侵对土壤氮有效性的影响程度和生理生态学机制。基于107篇相 关研究文献数据的整合, 发现植物入侵区域相对于无入侵区域土壤总氮、铵态氮、硝态氮、无机氮、微生物生物量氮含量显 著增加，增幅分别为 $(50 \pm 14) \% 、(60 \pm 24) \% 、(470 \pm 115) \% 、(69 \pm 25) \% 、(54 \pm 20) \%$ 。土壤硝态氮含量增幅较大反映硝化作 用增强, 这可能增加入侵植物硝态氮利用以及喜硝植物的共存。温带地区植物入侵后土壤的硝态氮含量增幅显著高于亚热带 地区。固氮植物入侵后土壤的总氮和无机氮含量增幅均显著高于非固氮植物入侵。木本和常绿植物入侵后土壤的总氮含量增 幅分别高于草本和落叶植物入侵; 而土壤铵态氮含量的增幅没有显著差异且与固氮入侵植物占比无明显关系; 然而硝态氮 含量的增幅普遍较高且与固氮入侵植物占比显著正相关。外来入侵植物固氮功能以及调落物质量和数量是影响土壤氮矿化和 硝化过程的关键因素。该研究为理解外来植物入侵成功和加剧的机制以及入侵植物功能性状与土壤氮动态之间的关系提供了 新的见解。
\end{abstract}

关键词 外来植物; 入侵生态学; 土壤氮循环; 氮有效性; 铵态氮; 硝态氮; 土壤总氮

许浩, 胡朝臣, 许士麒, 孙新超, 刘学炎 (2018). 外来植物入侵对土壤氮有效性的影响. 植物生态学报, 42, 1120-1130. DOI: 10.17521/cjpe.2018.0219

\section{Effects of exotic plant invasion on soil nitrogen availability}

\author{
XU Hao, HU Chao-Chen, XU Shi-Qi, SUN Xin-Chao, and LIU Xue-Yan*
}

Institute of Surface-Earth System Science, Tianjin University, Tianjin 300072, China

\begin{abstract}
Aims Exotic plant invasion has been a global eco-environmental issue, which declines biodiversity and influences ecosystem structure and function. Over the past decades, more and more studies showed that influences of exotic plant invasion on soil nitrogen $(\mathrm{N})$ cycles, and soil $\mathrm{N}$ availabilities can facilitate the success and aggravation of invading plants.

Methods Based on differences in soil $\mathrm{N}$ contents between invaded and uninvaded areas in natural ecosystems at the same study sites, this study explored magnitudes and ecophysiological mechanisms of soil $\mathrm{N}$ variations under exotic plant invasion.

Important findings Based on the data integrated from 107 papers, we found that contents of soil total N, ammonium-N, nitrate-N, inorganic $\mathrm{N}$, microbial biomass $\mathrm{N}$ under exotic plant invasion were increased by $(50 \pm 14) \%$, $(60 \pm 24) \%,(470 \pm 115) \%,(69 \pm 25) \%,(54 \pm 20) \%$ respectively relative to those under no invasion. The increment in the soil nitrate-N pool was highest, suggesting higher nitrification rate, which potentially promoted plant nitrate-N utilization and the coexistence of nitrate-preferring species. The increment of soil nitrate-N pool under invasion was higher in the temperate zone than the subtropical zones significantly. Invasion of $\mathrm{N}_{2}$-fixing plants caused obviously larger increments of soil total $\mathrm{N}$ and nitrate- $\mathrm{N}$ contents compared to invasion of non- $\mathrm{N}_{2}-$ fixing plants. The invasion of woody and evergreen invasive plants caused larger increments of soil total $\mathrm{N}$ than herbaceous and deciduous plants, respectively. The increases in soil ammonium-N under invasion did not differ substantially among different life forms and showed no clear relationship with the percentage of $\mathrm{N}_{2}$-fixing plants. Differently, soil nitrate-N showed much larger increments under invasion and showed positive linear relationship with the percentage of $\mathrm{N}_{2}$-fixing invasive plants. The $\mathrm{N}_{2}$-fixing function, litter quality and quantity of invasive plants are important factors regulating soil $\mathrm{N}$ mineralization and nitrification under invasion. This study provides

收稿日期Received: 2018-09-11 接受日期Accepted: 2018-10-18

基金项目: 国家自然科学基金项目(41730855、41522301和41473081)。Supported by the National Natural Science Foundation of China (41730855, 41522301 and 41473081).

* 通信作者Corresponding author (liuxueyan@tju.edu.cn)
\end{abstract}


novel insights into the mechanisms underlying the success and aggravation of plant invasion and into the relationships between soil $\mathrm{N}$ dynamics and plant functional traits in ecosystems under exotic plant invasion.

Key words exotic plant; invasion ecology; soil nitrogen cycle; nitrogen availability; ammonium; nitrate; soil total nitrogen

Xu H, Hu CC, Xu SQ, Sun XC, Liu XY (2018). Effects of exotic plant invasion on soil nitrogen availability. Chinese Journal of Plant Ecology, 42, 1120-1130. DOI: 10.17521/cjpe.2018.0219

外来植物入侵指在人为或自然因素作用下, 植 物由原产地进入新栖息地存活、繁殖、建群、扩张, 并造成明显的生态或经济影响的现象(Theoharides \& Dukes, 2007; Zheng et al., 2018)。植物入侵减少生 物多样性, 破坏生态系统结构和功能, 威胁农、林、 牧业发展, 造成巨大经济损失, 现已成为较严重的 生态环境问题(Pimentel et al., 2005; Ding et al., 2008; 类延宝等, 2010; 李博和马克平, 2010; 间小玲等, 2012)。

由于氮是植物生存生长重要的限制性资源, 因 此入侵植物如何影响土壤氮有效性是理解其入侵机 制的重要研究内容(Ehrenfeld, 2003; Sardans et al., 2016)。入侵条件下土壤氮有效性的变化直接关系到 入侵植物与本地植物的氮竞争关系和各自的氮利用 策略(Liao et al., 2008; Feng et al., 2009), 从而影响 外来植物的入侵程度和本地植物的消失或共存 $(\mathrm{Hu}$ et al., 2018)。目前, 大量野外和室内栽培实验发现 入侵区域与无入侵区域或入侵前后土壤不同形态氮 含量(Dreiss \& Volin, 2013; Cusack \& Mccleery, 2014)、氮转化过程(如矿化、氨氧化、硝化)速率 (Booth et al., 2003; Rossiter-Rachor et al., 2009)和相 关微生物 (如氨氧化细菌、硝化细菌) 群落丰度 (Hawkes et al., 2005; 于兴军等, 2005; Niu et al., 2007; Xiao et al., 2014)存在不同程度的差异, 为植 物入侵可改变土壤氮有效性的观点提供了充足证据 (Liao et al., 2008; Chen et al., 2009)。入侵后调落物 的质量和数量发生变化, 土壤氮转化过程中微生物 活性的改变等与入侵前存在差异是入侵植物影响土 壤氮有效性的重要机制(Lee et al., 2016; McLeod et al., 2016)(图1)。

对这些方面的研究结果和证据进行整合分析有 助于揭示植物入侵对土壤氮有效性影响的普遍规律 和机制(Gurevitch et al., 2018)。前人对野外和室内栽 培实验进行过整合分析, 揭示了植物入侵不同程度 地增加了土壤氮有效性(Liao et al., 2008; Vilà et al., 2011; Castro-Díez et al., 2014)。然而, Schittko等
(2016)发现室内栽培与野外控制实验的结果并不一 致, 自然入侵生态系统原位的土壤氮有效性变化幅 度、主控因子和机制并不十分清楚。此外, 植物的 入侵能力和地点的可入侵性共同决定着入侵后土壤 氮有效性的变化幅度(Milbau et al., 2003)(图1)。不同 气候带的入侵地点以及不同生活型和固氮功能的入 侵植物存在生物学特性和入侵地非生物因素等方面 的异质性(Liao et al., 2008)。因此很有必要对野外的 原位研究进行单独的整合分析。

基于以上研究现状, 本文详细归纳了同一研究 地外来植物入侵区域和无入侵区域的土壤原位氮含 量, 对比和分析不同气候带(温带、亚热带、热带) 的入侵地点以及不同生活型(一年生草本、多年生草 本、落叶灌木、常绿灌木、落叶乔木、常绿乔木、 多年生草质藤本、落叶木质藤本、常绿木质藤本) 和固氮功能(固氮、非固氮)的植物入侵影响土壤氮 指标的程度及其变化规律(图1), 探讨外来植物入侵 影响土壤氮转化过程和有效性的机制。主要目标为: (1)确定全球尺度下植物入侵对原位土壤氮有效性

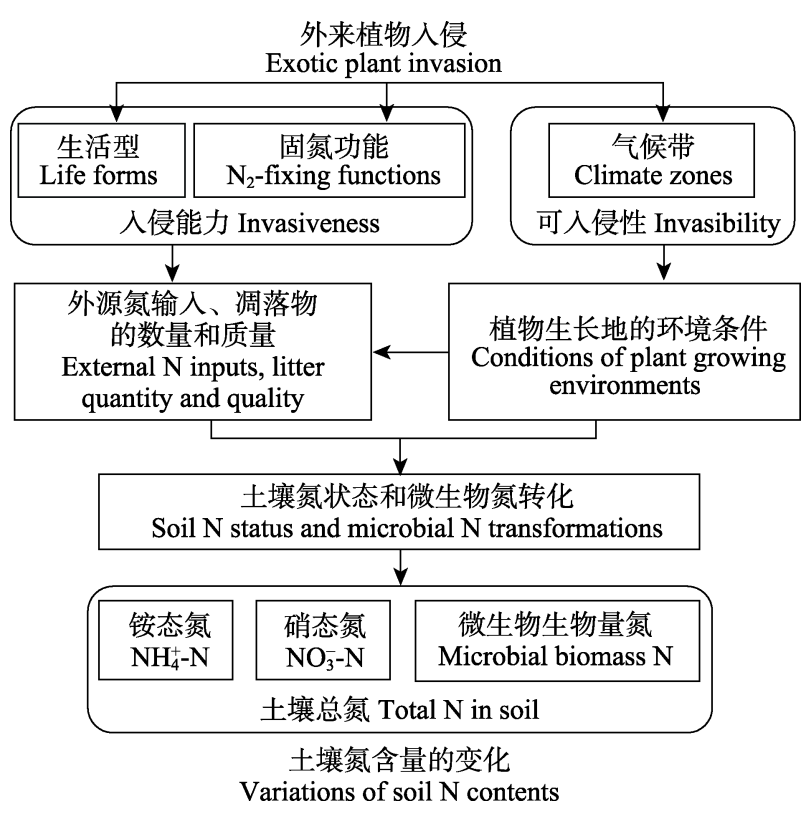

图1 外来植物入侵影响土壤氮(N)有效性的关键过程机制。 Fig. 1 Major processes and mechanisms of changing soil nitrogen $(\mathrm{N})$ availability influenced by exotic plant invasion. 
的影响趋势及具体程度; (2)确定不同气候带的入侵 地点以及不同生活型和固氮功能入侵植物的背景下, 植物入侵对土壤氮有效性的影响差异。

\section{1 数据来源和分析}

在Web of Science中通过关键词的单独和组合 搜索查找已发表研究, 所用关键词包括: invasive plant、plant invasion、encroachment、soil nitrogen、 soil nitrogen availability、soil available nitrogen、soil dissolved organic nitrogen、soil microbial biomass、 soil microbial nitrogen。为了获得在原位条件下外来 植物入侵对自然生态系统土壤氮有效性的影响, 我 们提取和整合的数据结果同时满足 3 个条件: (1)属 于自然陆地生态系统原位土壤的分析结果(排除培 养或控制实验、农业生态系统); (2)同时分析了同 一研究地点或样地内的入侵样方和无入侵样方 (二 者相距从几米到几百米不等)土壤氮含量; (3)入侵 和无入侵的土壤类型和环境地理条件无明显差异。 截止到2018年7月18日，按上述条件共笛选到107篇 文章, 具体采集的数据指标为入侵区域和无入侵区 域的土壤总氮、铵态氮、硝态氮、无机氮(铵态氮和 硝态氮之和)、微生物生物量氮含量。根据数据的可 获得性, 统计和做图分析统一为每个生态系统所获 得相应指标数据的平均值, 共有土壤总氮、铵态氮、 硝态氮、无机氮、微生物生物量氮含量数据分别为 $118 、 62 、 71 、 60 、 11$ 组(附件I)。采样地气候带(温 带、亚热带、热带)划分基于其纬度数据。入侵植物 的固氮和非固氮功能基于文献介绍和记录进行区分, 生活型(一年生草本、多年生草本、落叶灌木、常绿 灌木、落叶乔木、常绿乔木、多年生草质藤本、落 叶木质藤本、常绿木质藤本) 基于 Wikipedia (https://en.wikipedia.org/wiki/Main_Page)、Practical Plants (http://practical-plants.org/wiki/Practical_Plants) 和文献记录进行划分。

外来植物入侵区域相对于无入侵区域土壤氮指 标相对变化 $(R V$, 以 \%表示)的计算方法为:

$$
R V=\left(\left(C_{\mathrm{I}}-C_{\mathrm{N}}\right) / C_{\mathrm{N}}\right) \times 100 \text { 。 }
$$

其中, $C_{\mathrm{I}}$ 和 $C_{\mathrm{N}}$ 分别为入侵区域土壤和无入侵区 域土壤相同氮指标的值。参考Liao等(2008), 全组的 $R V$ 值显著高于、低于 $0.0 \%$ 时 (独立样本 $t$ 检验, 显著 水平 $p$ 设为 0.05 )表示外来植物入侵增加, 降低了对 应氮指标的值。不同气候带、固氮功能和生活型之
间相关指标的差异采用单因素方差分析(显著水平 $p$ 设为 0.05$)$ 。土壤铵态氮含量的增幅与固氮入侵植物 占比(特定生活型入侵植物中, 可固氮的入侵植物 株数(非物种数)的比例)、土壤硝态氮含量增幅之间 的相关性采用非线性拟合分析，土壤硝态氮含量的 增幅与固氮入侵植物占比之间的相关性采用线性拟 合分析。统计分析采用SPSS 25.0软件。土壤氮指标 值及其 $R V$ 值均表达为平均值(标准误差, $S E$ )。

\section{2 结果和讨论}

\section{1 植物入侵区域与无入侵区域的土壤氮含量差异}

根据整合的数据，入侵区域土壤的总氮含量范 围为 $0.0 \%-2.7 \%$, 平均值为 $(0.4 \pm 0.0) \%$ (图 $2 \mathrm{~A}$ ); 无 入侵区域土壤的总氮含量范围为 $0.0 \%-1.9 \%$, 平均 值为 $(0.3 \pm 0.0) \%$ (图 $2 \mathrm{~A}$ )。入侵区域相对于无入侵区 域土壤总氮含量显著增加 $(p<0.05), R V$ 值为 $(50 \pm$ $14) \%$ (图2B)。从入侵植物自身的因素来看, 很多外 来入侵植物具有固氮功能, 入侵植物本身会增加土 壤的氮输入(Caldwell, 2006; González-Muñoz et al., 2012)。而且, 入侵植物通常根系更为发达, 能够获 取和利用深层土壤氮源, 并通过调落物将其归还到 表层土壤(Li et al., 2007; Broadbent et al., 2017), 这 种“氮泵”效应是增加入侵地表层土壤总氮含量的原 因之一(Wei et al., 2017)。从土壤微生物因素来看, 入侵植物的生产力普遍高于本地植物, 导致入侵区 域土壤有机碳、氮输入增加(van Kleunen et al., 2010; Godoy et al., 2011), 这会增加土壤的非共生固氮量 (Zhang et al., 2010; Xu et al., 2012)。此外, 入侵区域 土壤的微生物生物量氮含量总体也高于无入侵区域 (图2), 氮固持能力的增加一定程度上减少了土壤氮 淋溶丢失(Li et al., 2007)。

入侵区域土壤的无机氮含量范围为1.9-143.1 $\mathrm{mg} \cdot \mathrm{kg}^{-1}$, 平均值为 $(19.3 \pm 3.7) \mathrm{mg} \cdot \mathrm{kg}^{-1}$ (图2A); 无 入侵区域土壤的无机氮含量范围为 $0.7-165.5$ $\mathrm{mg} \cdot \mathrm{kg}^{-1}$, 平均值为 $(15.1 \pm 3.4) \mathrm{mg} \cdot \mathrm{kg}^{-1}$ (图2A)。其中, 入侵区域土壤的铵态氮、硝态氮含量范围分别为 1.4-123.6 $\mathrm{mg} \cdot \mathrm{kg}^{-1}$ 和 $0.1-57.2 \mathrm{mg} \cdot \mathrm{kg}^{-1}$, 平均值分别 为 $(13.0 \pm 2.8) \mathrm{mg} \cdot \mathrm{kg}^{-1}$ 和 $(6.5 \pm 1.3) \mathrm{mg} \cdot \mathrm{kg}^{-1}$ (图2A)。 无入侵区域土壤的铵态氮、硝态氮含量范围分别为 0.6-157.5 $\mathrm{mg} \cdot \mathrm{kg}^{-1}$ 和0.0-51.9 $\mathrm{mg} \cdot \mathrm{kg}^{-1}$, 平均值分别 为 $(10.6 \pm 2.7)$ 和 $(3.8 \pm 1.1) \mathrm{mg} \cdot \mathrm{kg}^{-1}$ (图 $2 \mathrm{~A}$ )。相对于 无入侵区域，入侵区域土壤的无机氮、铵态氮和硝 


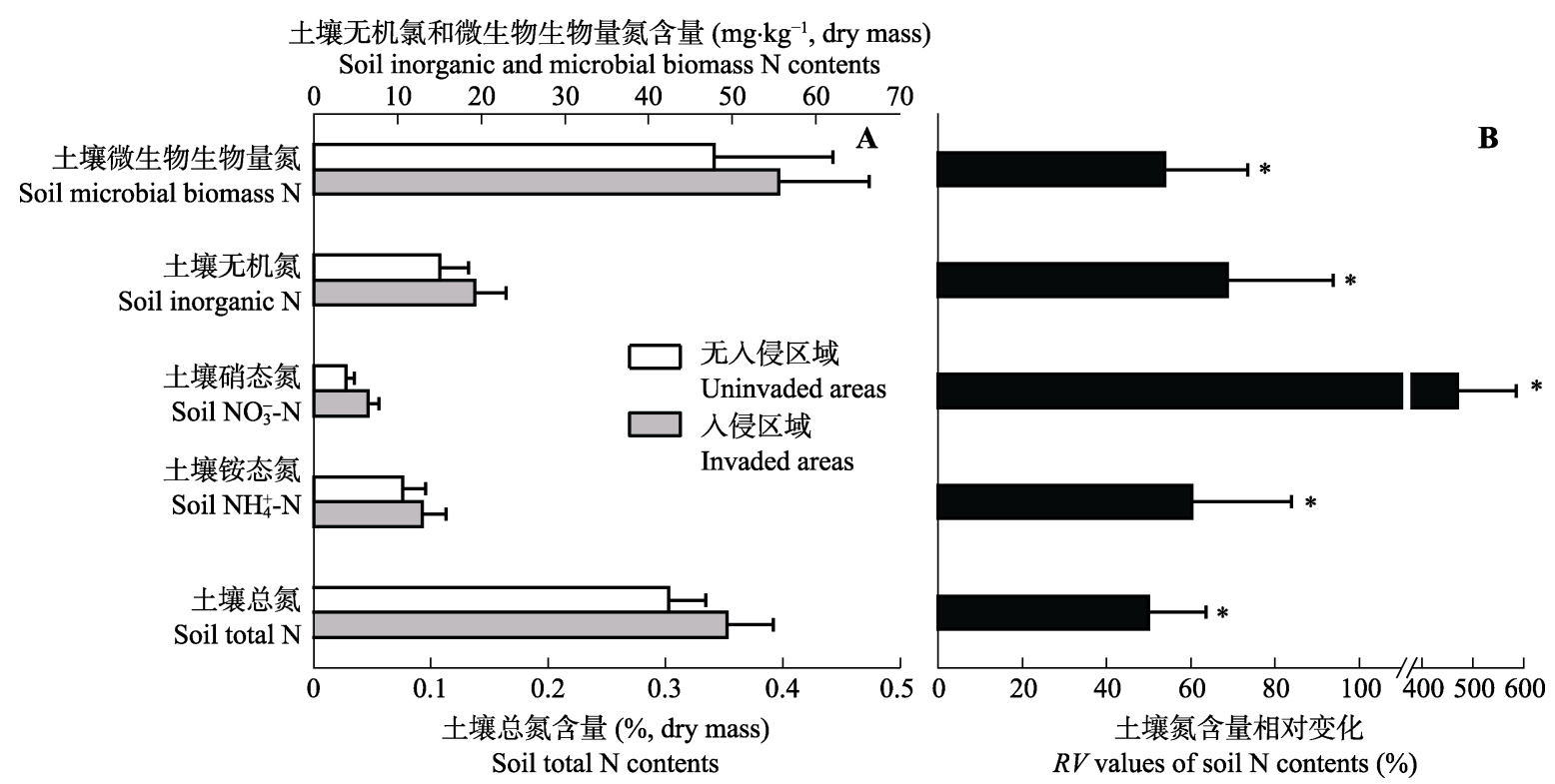

图2入侵区域与无入侵区域土壤的氮含量(A)和入侵区域相对于无入侵区域土壤氮含量的相对变化(B) (平均值+标准误差)。 *表示入侵区域相对于无入侵区域土壤氮含量变化显著 $(p<0.05)$ 。

Fig. 2 Soil $\mathrm{N}$ contents in invaded and uninvaded areas (A) and relative variation $(R V)$ values of soil $\mathrm{N}$ contents in invaded areas compared to uninvaded areas (B) (mean $+S E)$. * indicates significant variation of soil $\mathrm{N}$ contents between the two types of areas $(p<$ $0.05)$.

态氮含量显著增加 $(p<0.05), R V$ 值分别为 $(69 \pm$ $25) \% 、(60 \pm 24) \%$ 和 $(470 \pm 115) \%$ (图2B)，显示外来 植物入侵增加了土壤氮有效性。根据上述结果, 入 侵区域和无入侵区域土壤无机氮均以铵态氮为主, 表明植物入侵后土壤铵态氮占主导的化学结构特征 未被改变(图2A)。此外, 入侵对土壤无机氮和占优 势的铵态氮含量的影响幅度也基本和总氮的增加幅 度相当(图2A)。一方面, 固氮入侵植物和被促进的 土壤微生物非共生固氮能够直接增加土壤铵态氮含 量(González-Muñoz et al., 2012; Xu et al., 2012)。另 一方面, 入侵植物调落物普遍具有较高的氮含量、 较低的碳氮比(C:N)和木质素氮比(lignin: $\mathrm{N})($ Liao et al., 2008; Castro-Díez et al., 2014)，同时入侵区域的 土壤有机质分解速率(Strickland et al., 2010)与碳氮 循环酶的活性更高(Marchante et al., 2008; Sun et al., 2013; Souza-Alonso et al., 2014), 更快的土壤有机 质矿化速率提高了土壤铵态氮的含量 (Rossiterachor et al., 2009)。然而, 我们发现土壤硝态氮含量 的增幅远高于无机氮和铵态氮的增幅, 其平均含量 增加的幅度是铵态氮的近8倍(图2B)。这表明外来植 物入侵对土壤硝化作用的促进更大, 尤其相对于硝 态氮丢失途径(反硝化和淋溶过程), 因此造成比例 上硝态氮含量的增加更为显著。机制上, 氨氧化细 菌和硝化细菌是硝态氮生产的主导者, 其丰度在很
多外来入侵地被发现明显增加(Shannon- Firestone et al., 2015; McLeod et al., 2016)。主要原因是入侵后 土壤矿化作用的增强产生足够的铵态氮(Liao et al., 2008), 直接增加了在自养硝化细菌转化作用下的 硝态氮的产生(Cameron et al., 2012; Yang et al., 2017)。此外, 入侵植物根系发达, 能够增加土壤通 气性并一定程度地抑制反硝化作用(Dassonville et al., 2011; Keser et al., 2015)。甚至, 研究发现入侵植 物净初级生产力与原位土壤硝态氮水平存在显著正 相关性(McLeod et al., 2016), 这可能是因为微生物 对土壤还原态氮的竞争能力高于植物, 使得入侵植 物被动地或者生理策略上主动地具有更高的硝态氮 吸收能力或偏好(Kourtev et al., 1999; Bloom, 2015), 这也是外来植物入侵区域土壤硝态氮含量增幅较大 的原因之一。

\section{2 不同气候带植物入侵区域和无入侵区域的土} 壤氮含量差异

除了外来植物的入侵能力, 入侵地点的环境可 入侵性(如土壤氮有效性水平)也是决定入侵程度和 扩张速度的重要原因(Milbau et al., 2003)。不同气候 带具有不同的温度和水分条件, 这是影响土壤氮转 化过程和氮有效性的重要环境因素(Reich \& Oleksyn, 2004; Wang et al., 2006)。本研究中, 温带、亚 热带、热带地区植物入侵区域土壤总氮含量的平均 
值分别为 $(0.4 \pm 0.0) \% 、(0.2 \pm 0.1) \% 、(0.4 \pm 0.2) \%$ (图 $3 \mathrm{~A})$; 无入侵区域土壤总氮含量的平均值分别为 $(0.3$ $\pm 0.0) \% 、(0.2 \pm 0.1) \% 、(0.3 \pm 0.1) \%$ (图3A)。其中，温 带、亚热带、热带地区入侵区域相对于无入侵区域 土壤总氮含量均显著增加 $(p<0.05), R V$ 值有所差异, 分别为 $(27.8 \pm 10.6) \%$ 、 $(82.1 \pm 38.2) \%$ 、 $(72.3 \pm 28.0) \%$ (图3E)。亚热带和热带地区入侵区域土壤总氮含量 的增幅高于温带地区, 这可能与热带、亚热带地区 的入侵植物生长速率更快(van Kleunen et al., 2010), 从而引发更为显著的 “氮泵”效应有关。

温带、亚热带、热带地区植物入侵区域相对于 无入侵区域土壤铵态氮含量的 $R V$ 值分别为 $(70.5 \pm$ $42.9) \% 、(57.4 \pm 24.6) \% 、(11.5 \pm 11.6) \%$ (图3F), 硝
态氮含量的 $R V$ 值分别为 $(683.0 \pm 190.4) \%$ 、(183.6 \pm $91.5) \% 、(492.5 \pm 440.1) \%$ (图3G)。对比发现，除了 热带地区入侵区域和无入侵区域土壤的铵态氮含量 无显著差异, 其他气候带的入侵区域相对于无入侵 区域的土壤铵态氮和硝态氮含量均显著增加 $(p<$ 0.05)。热带地区土壤氮循环更开放, 较快的土壤氨 化作用(Martinelli et al., 1999)和入侵植物对铵态氮 的偏好性吸收(Hu et al., 2018)可能导致入侵区域土 壤铵态氮含量的增幅减少, 因此与无入侵差异不显 著。总体上, 温带地区入侵区域土壤铵态氮和硝态 氮含量的增幅高于亚热带和热带地区(图3F、3G)。 相对而言, 温带地区较低的气温和降水量条件使得 生态系统土壤无机氮水平通常较低且植物生长处于
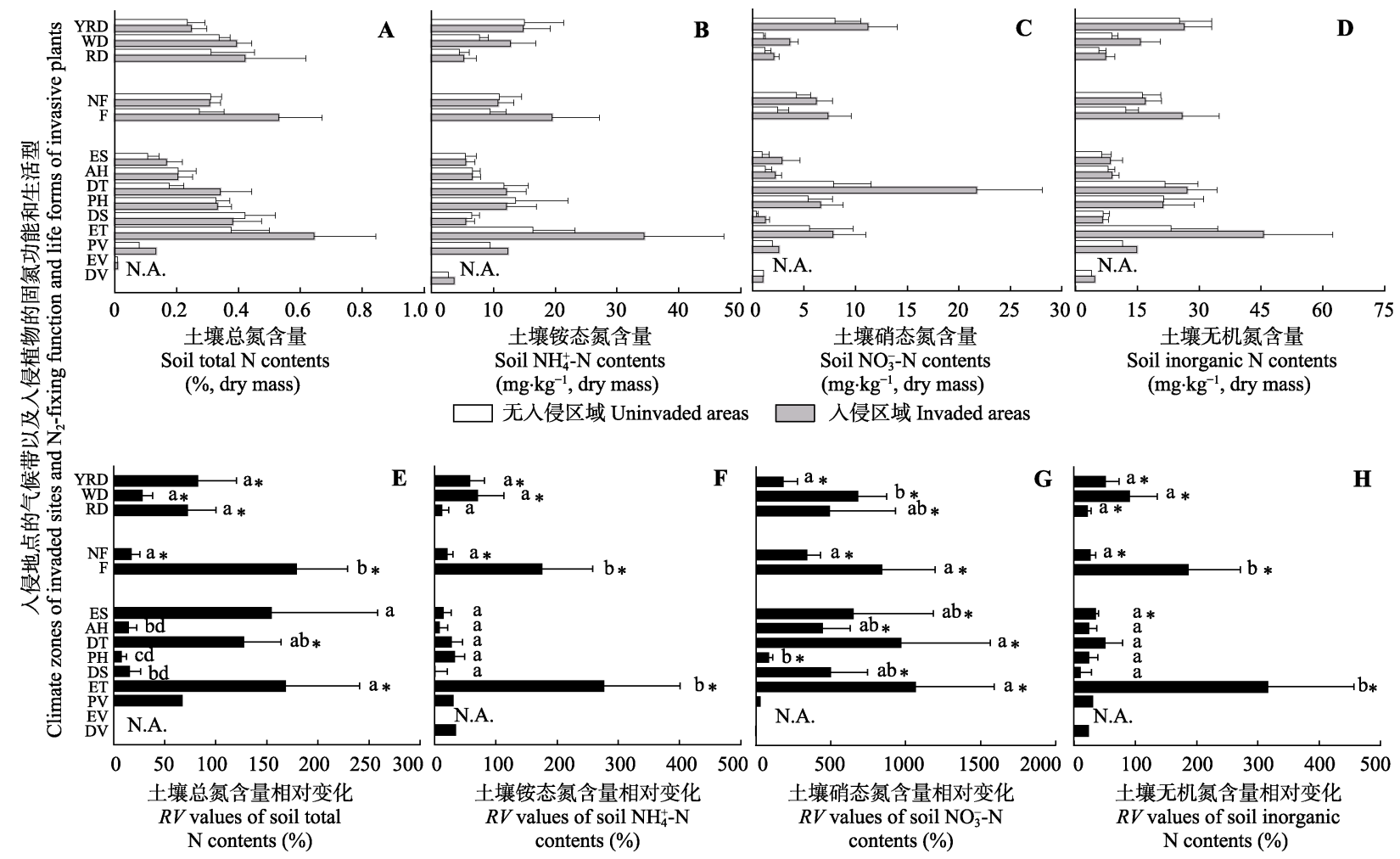

$\square$ 无人侵区域 Uninvaded area

$\square$ 人侵区域 Invaded areas
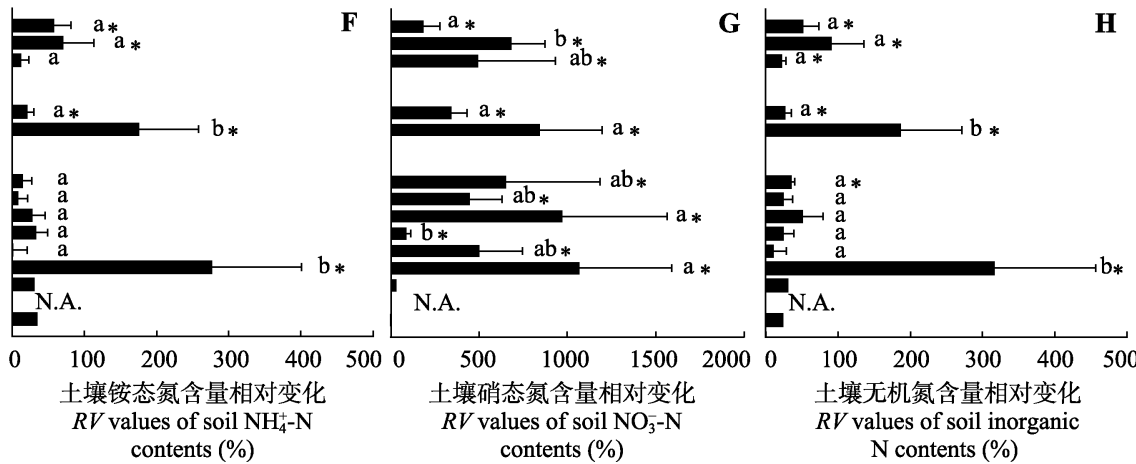

图3 不同气候带、固氮功能、生活型分类下，入侵区域和无入侵区域土壤的总氮 $(\mathbf{A})$ 、铵态氮 $(\mathbf{B})$ 、硝态氮 $(\mathbf{C})$ 、无机氮含量 $(\mathbf{D})$ 和入侵区域相对于无入侵区域土壤总氮 $(\mathbf{E}) 、$ 铵态氮 $(\mathbf{F}) 、$ 硝态氮 $(\mathbf{G})$ 、无机氮 $(\mathbf{H})$ 含量的相对变化(平均值+标准误差)。温带、 亚热带、热带分别缩写为WD、YRD、RD; 固氮、非固氮分别缩写为F、NF; 一年生草本、多年生草本、常绿灌木、落叶灌 木、常绿乔木、落叶乔木、多年生草质藤本、落叶木质藤本、常绿木质藤本分别缩写为AH、PH、ES、DS、ET、DT、PV、 DV、EV。N.A.表示没有数据; 图 $\mathbf{E}-\mathbf{H}$ 中不同小写字母表示差异显著性 $(p<0.05)$; *表示入侵区域相对于无入侵区域土壤氮含 量变化显著 $(p<0.05)$ 。

Fig. 3 Soil total $\mathrm{N}(\mathbf{A}), \mathrm{NH}_{4}^{+}-\mathrm{N}(\mathbf{B}), \mathrm{NO}_{3}^{-}-\mathrm{N}(\mathbf{C})$, inorganic $\mathrm{N}(\mathbf{D})$ contents in invaded and uninvaded areas and relative variation $(R V)$ values of soil total $\mathrm{N}(\mathbf{E}), \mathrm{NH}_{4}^{+}-\mathrm{N}(\mathbf{F}), \mathrm{NO}_{3}^{-}-\mathrm{N}(\mathbf{G})$, inorganic $\mathrm{N}(\mathbf{H})$ contents in invaded areas compared to uninvaded areas among different climate zones, invasive plants with different $\mathrm{N}_{2}$-fixing functions and life forms (mean $+S E$ ). WD, YRD, and RD represent temperate, subtropical, and tropical zones, respectively; $\mathrm{F}$ and NF represent invasive plants with $\mathrm{N}_{2}$-fixing and non- $\mathrm{N}_{2}$-fixing functions, respectively; and AH, PH, ES, DS, ET, DT, PV, DV, and EV represent annual herb, perennial herb, evergreen shrub, deciduous shrub, evergreen tree, deciduous tree, perennial vine, deciduous vine, and evergreen vine, respectively. N.A. denotes data not available, and different letters in panels $\mathbf{E}-\mathbf{H}$ indicate significant differences $(p<0.05)$ while * indicates significant variation of soil $\mathrm{N}$ contents in invaded areas compared to uninvaded areas $(p<0.05)$. 
氮限制状态(Martinelli et al., 1999; Tang et al., 2017)。我们的结果表明外来植物入侵增加了土壤的 无机氮含量, 这在氮矿化、硝化过程原本受非生物 因素限制的生态系统或地区更加明显(图3G)。前人 对比不同养分条件下植物入侵对土壤氮循环的影响, 同样发现外来植物入侵对低氮生态系统土壤氮有效 性的增幅更大(Sardans et al., 2016)。

\section{3 不同固氮功能、生活型的植物入侵区域和无入 侵区域的土壤氮含量差异}

探讨不同固氮功能和生活型入侵植物影响土壤 氮有效性的差异有助于更好地理解不同外来植物入 侵能力的差异性和土壤氮循环对植物入侵的反馈机 制(Peña et al., 2010)。植物固氮, 即大气 $\mathrm{N}_{2}$ 被根瘤菌 还原为氨并被植物利用的过程(Vitousek et al., 2002), 是陆地生态系统氮输入的重要途径, 也是固氮植物 生理生态学功能发挥的重要驱动力(Baer et al., 2006)。本研究中, 固氮和非固氮植物入侵区域相对 于无入侵区域土壤总氮含量的 $R V$ 值分别为 $(178.7 \pm$ $50.6) \%$ 和 $(17.2 \pm 8.7) \%$ (图3E), 铵态氮含量的 $R V$ 值 分别为 $(174.9 \pm 83.0) \%$ 和 $(20.3 \pm 9.6) \%$ (图3F), 硝态 氮含量的 $R V$ 值分别为 $(842.8 \pm 354.8) \%$ 和 $(340.5 \pm$ $0.0) \%$ (图3G)。固氮和非固氮植物入侵区域相对于 无入侵区域土壤总氮、铵态氮、硝态氮、无机氮含 量均显著增加 $(p<0.05)$, 但是固氮植物入侵区域土 壤总氮、铵态氮、无机氮含量的增幅显著高于非固 氮植物入侵 $(p<0.05)$ (图3)。我们的结果清楚地揭示 了固氮功能是入侵植物驱动土壤总氮库增加以及提 高氮矿化、硝化作用的重要原因。一方面, 固氮植 物入侵直接增加土壤氮输入(Galloway et al., 2004; Baer et al., 2006)的同时也一定程度减少植物群落生 长的氮限制; 另一方面, 固氮植物调落物氮含量较 高, 土壤有机质分解与氮矿化速率也较高(Allison et al., 2006; Perakis \& Hibbs, 2014)。

不同生活型的入侵植物存在光合、生长速率、 氮吸收能力和策略等诸多功能性状差异(Liao et al., 2008), 其调落物质量和数量的差异直接关系到土 壤有机质积累和分解, 碳氮循环微生物过程、速率 等方面, 从而影响土壤的氮有效性(Yé et al., 2017)。 该研究中, 一年生草本、多年生草本、落叶灌木、 常绿灌木、落叶乔木、常绿乔木植物入侵区域相对 于无入侵区域土壤总氮含量的 $R V$ 值分别为 $(14.2 \pm$
$8.5) \% 、(7.6 \pm 4.7) \% 、(15.1 \pm 11.4) \% 、(154.6 \pm$ $103.8) \% 、(127.0 \pm 37.1) \%$ 、(168.3 \pm 72.7$) \%$ (图3E)。 对比 $R V$ 值发现, 落叶和常绿乔木植物入侵区域相对 于无入侵区域土壤总氮含量的增幅总体较高，而这 两类生活型入侵植物的固氮植物占比(分别为 $75 \%$ 和 $59 \%$ ) 高于其余 4 种生活型(分别为 $0 \% 、 13 \% 、 14 \%$ 、 $29 \%$ )。另外, 从相对于无入侵区域土壤总氮含量增 幅的数值看, 木本入侵植物高于草本入侵植物, 常 绿入侵植物高于落叶入侵植物(图3E)。其中, 木本 入侵植物中的固氮植物占比为 $44 \%$; 草本入侵植物 中的固氮植物占比为 $6 \%$; 常绿入侵植物中的固氮 植物占比为 $37 \%$; 而落叶入侵植物中的固氮植物占 比为 $52 \%$ 。乔木植物入侵区域土壤总氮含量的增幅 较大, 木本植物入侵对土壤总氮含量的增幅高于草 本植物, 这主要归因于固氮植物增加氮输入和土壤 总氮的积累。而常绿和落叶两种生活型入侵植物的 固氮植物占比较为接近, 这主要反映常绿植物调落 物的分解速率慢于落叶植物, 氮矿化和丢失总体较 低, 因此其入侵区域土壤总氮含量的增幅较大(Liao et al., 2006; Polyakova \& Billor, 2008; Solly et al., 2014)。这一机制同样支持木本植物相对于草本植物 入侵区域土壤总氮含量增幅较大的结果。

一年生草本、多年生草本、落叶灌木、常绿灌 木、落叶乔木、常绿乔木植物入侵区域相对于无入 侵区域土壤铵态氮含量的 $R V$ 值分别为 $(8.1 \pm$ $13.8) \% 、(32.7 \pm 16.4) \% 、(1.0 \pm 19.5) \% 、(14.2 \pm$ $13.1) \% 、(26.6 \pm 19.0) \% 、(275.6 \pm 125.2) \%$ (图3F), 硝 态氮含量的 $R V$ 值分别为 $(447.4 \pm 180.7) \%$ 、(85.3 \pm $24.6) \% 、(500.6 \pm 243.9) \% 、(652.8 \pm 553.3) \%$ 、 $(971.0$ $\pm 595.0) \%$ 、 $(1066.8 \pm 525.3) \%$ (图3G)。不同生活型 植物间凋落物质量差异是调控土壤氮循环和无机氮 含量的重要植物因素(Jiang et al., 2013)。植物调落 物和土壤有机质中的氮矿化是土壤铵态氮产生的主 要途径(Knops et al., 2002; Chapman et al., 2006), 随 后土壤铵/氨在硝化细菌的氧化下生成硝态氮(Yang et al., 2017)。在多数陆地生态系统中, 土壤氮矿化 和硝化、铵态氮和硝态氮含量之间存在正相关关系 (Vitousek \& Matson, 1984; Chapin III et al., 2011)。因 此, 不同生活型植物入侵后土壤铵态氮和硝态氮含 量可能同时增加。如果它们的增幅之间存在正相关 关系, 反映了特定生活型植物入侵同步提高土壤氮 
矿化和硝化过程。然而, 该研究中, 只有常绿乔木植 物入侵区域土壤的铵态氮含量显著增加且高于其他 生活型 $(p<0.05)$, 其余5种生活型植物入侵区域相 对于无入侵区域, 土壤铵态氮含量的增幅较小且相 互间差异不显著(图3F)。对硝态氮而言，所有生活型 的入侵植物均显著增加了土壤硝态氮含量 $(p<$ 0.05)(图3G)。其中, 木本植物入侵后土壤硝态氮含 量的增幅总体高于草本植物入侵，常绿植物入侵后 土壤硝态氮含量的增幅总体高于落叶植物入侵(图 $3 \mathrm{G})$ 。不同生活型植物入侵对土壤硝态氮含量的影 响明显强于对铵态氮含量的影响, 且二者的增幅不 存在明显关系(图4A)。相应土壤铵态氮和硝态氮含 量增幅的变化特征不能从不同生活型植物之间(如 落叶与常绿、草本和木本) 调落物数量和质量的特异 性所造成的分解差异得到解释(Liao et al., 2006; Polyakova \& Billor, 2008; Solly et al., 2014)。

当前, 在入侵生态学和生物地球化学研究中, 探讨入侵植物功能特征与其对土壤氮循环影响幅度 之间详细关系的研究还非常稀少, 其机制也函待研 究(Liao et al., 2008)。除了调落物分解和有机氮矿化, 入侵植物根系固氮(即根瘤菌将大气 $\mathrm{N}_{2}$ 转化为 $\mathrm{NH}_{3}$
的过程; Bloom, 2015)可能是控制入侵区域土壤铵 态氮和硝态氮含量增加趋势和幅度的另一重要植物 学因素(Baer et al., 2006)。该研究中, 我们发现入侵 区域土壤铵态氮含量的增幅随固氮入侵植物占比无 明显增加关系(图4A), 而硝态氮含量的增幅随固氮 入侵植物占比增加呈线性增加 $\left(R^{2}=0.86, p<0.01\right.$; 图4B)。在自养硝化作用中，作为反应底物的氨，其 含量是硝态氮生产速率的主要限制因素(Yang et al., 2017)。铵/氨的可利用性或丰度增加会增加氨氧化 细菌丰度并提高硝化速率(Lu et al., 2015; Shannon-Firestone et al., 2015)。入侵植物根系固氮作用 增加将直接增加硝化细菌的氨可利用性，这可能是 本研究中土壤铵态氮含量增幅小且与硝态氮含量增 幅、固氮入侵植物占比关系不明显的主要原因。然 而，入侵植物固氮功能及其如何通过地上-地下生 理生态学过程影响土壤氮矿化和硝化的详细机制和 证据还有待进一步研究。

\section{3 结论}

本研究聚焦陆地生态系统外来植物入侵对土壤 原位氮含量的影响幅度和机制。主要规律为: 植物

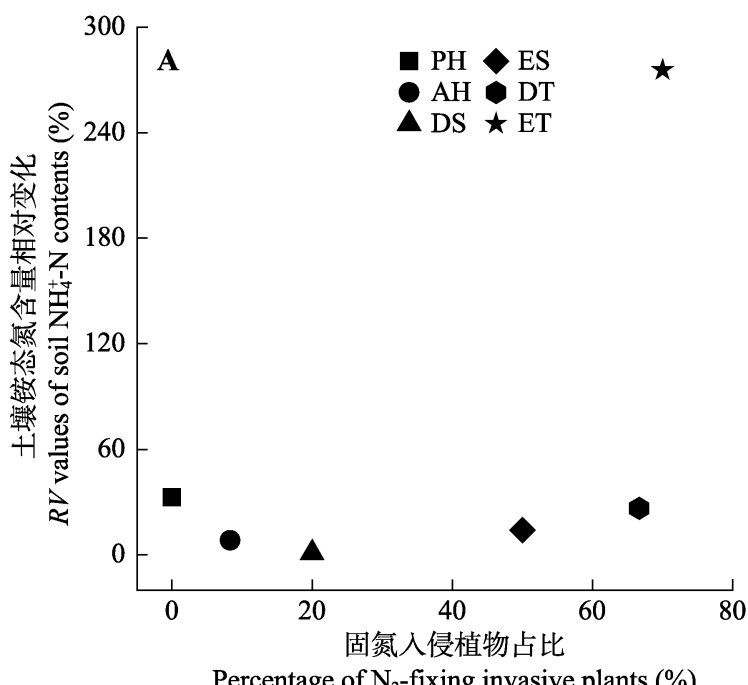

Percentage of $\mathrm{N}_{2}$-fixing invasive plants (\%)

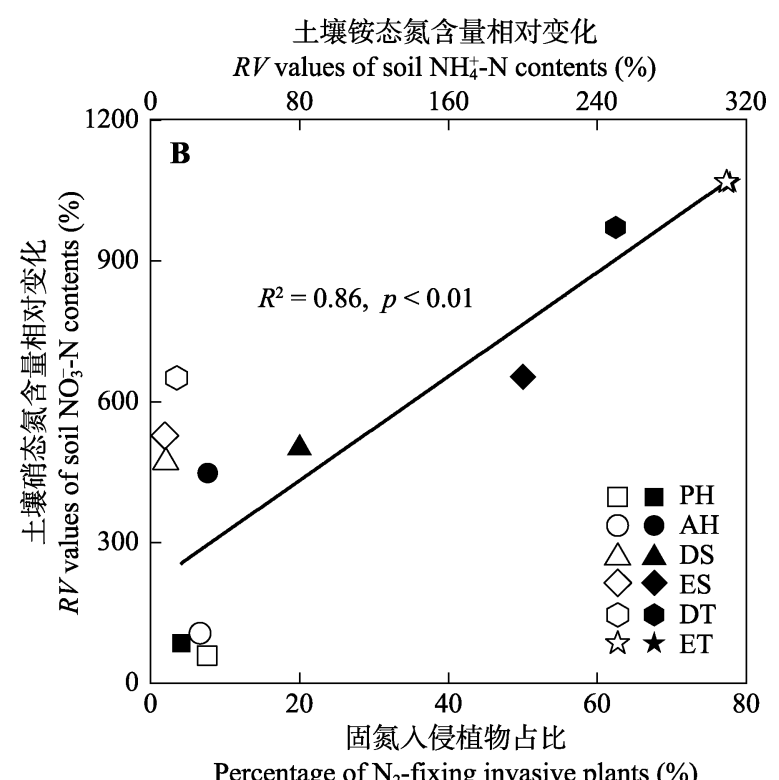

Percentage of $\mathrm{N}_{2}$-fixing invasive plants (\%)

图4 不同生活型植物入侵区域相对于无入侵区域土壤铵态氮含量的相对变化与固氮入侵植物占比之间的关系(A)和土壤硝 态氮含量的相对变化分别与铵态氮含量(空心符号)的相对变化、固氮入侵植物占比(实心符号)之间的关系(B)。一年生草本、 多年生草本、常绿灌木、落叶灌木、常绿乔木、落叶乔木分别缩写为AH、PH、ES、DS、ET、DT。

Fig. 4 Relationship between relative variation $(R V)$ values of soil $\mathrm{NH}_{4}^{+}-\mathrm{N}$ contents and percentage of $\mathrm{N}_{2}$-fixing invasive plants (A) and relationships between relative variation $(R V)$ values of soil $\mathrm{NO}_{3}^{-}-\mathrm{N}$ contents and those of soil $\mathrm{NH}_{4}^{+}-\mathrm{N}$ contents (hollow symbol), and percentage of $\mathrm{N}_{2}$-fixing invasive plants (solid symbol) (B) among different groups of invasive plants. AH, PH, ES, DS, ET, and DT represent annual herb, perennial herb, evergreen shrub, deciduous shrub, evergreen tree, and deciduous tree, respectively. 
入侵显著增加了土壤总氮、铵态氮、硝态氮、微生 物生物量氮含量, 但它们的相对变化存在差异, 尤 其土壤硝态氮含量增幅高达 $470 \%$ 。分类对比发现, 植物入侵对温带地区土壤硝态氮含量的影响高于亚 热带地区; 固氮植物入侵对土壤总氮、铵态氮和无 机氮含量的影响高于非固氮植物; 木本植物和常绿 植物入侵后土壤总氮的积累高于草本和落叶植物; 然而, 不同生活型植物入侵后土壤铵态氮含量的增 幅均低于土壤硝态氮, 且发现土壤硝态氮含量的增 幅随固氮入侵植物占比增加呈线性增长, 这些结果 清晰地揭示了植物固氮功能对土壤氮有效性和循环 过程机制的影响, 但其内部过程原理, 尤其二者之 间的生物学反馈机制有待进一步研究。该研究为理 解外来植物入侵成功和加剧的机制, 以及入侵生态 系统氮生物地球化学循环与环境和植物功能之间的 关系提供了新的见解。

致谢 感谢天津大学的宋韦讲师和董玉平博士在数 据分析、讨论中给予的指导。

\section{参考文献}

Allison SD, Nielsen C, Hughes RF (2006). Elevated enzyme activities in soils under the invasive nitrogen-fixing tree Falcataria moluccana. Soil Biology and Biochemistry, 38, 1537-1544.

Baer SG, Church JM, Williard KWJ, Groninger JW (2006). Changes in intrasystem $\mathrm{N}$ cycling from $\mathrm{N}_{2}$-fixing shrub encroachment in grassland: Multiple positive feedbacks. Agriculture Ecosystems and Environment, 115, 174-182.

Bloom AJ (2015). The increasing importance of distinguishing among plant nitrogen sources. Current Opinion in Plant Biology, 25, 10-16.

Booth MS, Stark JM, Caldwell MM (2003). Inorganic N turnover and availability in annual- and perennial-dominated soils in a northern Utah shrub-steppe ecosystem. Biogeochemistry, 66, 311-330.

Broadbent A, Stevens CJ, Peltzer DA, Ostle NJ, Orwin KH (2017). Belowground competition drives invasive plant impact on native species regardless of nitrogen availability. Oecologia, 186, 577-587.

Caldwell BA (2006). Effects of invasive scotch broom on soil properties in a Pacific coastal praire soil. Applied Soil Ecology, 32, 149-152.

Cameron KC, Di HJ, Moir JL (2012). Nitrogen losses from the soil-plant system: A review. Annals of Applied Biology,
$162,145-173$.

Castro-Díez P, Godoy O, Alonso A, Gallardo A, Saldana A (2014). What explains variation in the impacts of exotic plant invasions on the nitrogen cycle? A meta-analysis. Ecology Letters, 17, 1-12.

Chapin III FS, Matson PA, Vitousek PM (2011). Principles of Terrestrial Ecosystem Ecology. 2nd edn. Springer, New York.

Chapman SK, Langley JA, Hart SC, Koch GW (2006). Plants actively control nitrogen cycling: Uncorking the microbial bottleneck. New Phytologist, 169, 27-34.

Chen BM, Peng SL, Ni GY (2009). Effects of the invasive plant Mikania micrantha H.B.K. on soil nitrogen availability through allelopathy in South China. Biological Invasions, 11, 1291-1299.

Cusack DF, Mccleery TL (2014). Patterns in understory woody diversity and soil nitrogen across native- and non-nativeurban tropical forests. Forest Ecology and Management, 318, 34-43.

Dassonville N, Guillaumaud N, Piola F, Meerts P, Poly F (2011). Niche construction by the invasive Asian knotweeds (species complex Fallopia): Impact on activity, abundance and community structure of denitrifiers and nitrifiers. Biological Invasions, 13, 1115-1133.

Ding JQ, Mack RN, Lu P, Ren MX, Huang HW (2008). China's booming economy is sparking and accelerating biological invasions. BioScience, 58, 317-324.

Dreiss L, Volin JC (2013). Influence of leaf phenology and site nitrogen on invasive species establishment in temperate deciduous forest understories. Forest Ecology and Management, 296, 1-8.

Ehrenfeld JG (2003). Effects of exotic plant invasions on soil nutrient cycling processes. Ecosystems, 6, 503-523.

Feng YL, Lei YB, Wang RF, Callaway RM, Valiente-Banuet A, Inderjit, Li YP, Zheng YL (2009). Evolutionary tradeoffs for nitrogen allocation to photosynthesis versus cell walls in an invasive plant. Proceedings of the National Academy of Sciences of the United States of America, 106, 1853-1856.

Galloway JN, Dentener FJ, Capone DG, Boyer EW, Howarth RW, Seitzinger SP, Asner GP, Cleveland CC, Green PA, Holland EA, Karl DM, Michaels AF, Porter JH, Townsend AR, Vorosmarty CJ (2004). Nitrogen cycles: Past, present, and future. Biogeochemistry, 70, 153-226.

Godoy O, Valladares F, Castrodíez P (2011). Multispecies comparison reveals that invasive and native plants differ in their traits but not in their plasticity. Functional Ecology, 
$25,1248-1259$.

González-Muñoz N, Costa-Tenorio N, Espigares T (2012). Invasion of alien Acacia dealbata on Spanish Quercus robur forests: Impact on soils and vegetation. Forest Ecology and Management, 269, 214-221.

Gurevitch J, Koricheva J, Nakagawa S, Stewart G (2018). Meta-analysis and the science of research synthesis. $\mathrm{Na}$ ture, 555, 175-182.

Hawkes CV, Wren IF, Herman DJ, Firestone MK (2005). Plant invasion alters nitrogen cycling by modifying the soil nitrifying community. Ecology Letters, 8, 976-985.

Hu CC, Lei YB, Tan YH, Sun XC, Xu H, Liu CQ, Liu XY (2018). Plant nitrogen and phosphorus utilization under invasive pressure in a montane ecosystem of tropical China. Journal of Ecology, DOI: 10.1111/1365-2745. 13008.

Jiang J, Li YK, Wang MZ, Zhou CP, Cao GM, Shi PL, Song MH (2013). Litter species traits, but not richness, contribute to carbon and nitrogen dynamics in an alpine meadow on the Tibetan Plateau. Plant and Soil, 373, 931-941.

Keser LH, Visser EJW, Dawson W, Song YB, Yu FH, Fischer M, Dong M, van Kleunen M (2015). Herbaceous plant species invading natural areas tend to have stronger adaptive root foraging than other naturalized species. Frontiers in Plant Science, 6, 273. DOI: 10.3389/fpls.2015.00273

Knops JMH, Bradley KL, Wedin DA (2002). Mechanisms of plant species impacts on ecosystem nitrogen cycling. Ecology Letters, 5, 454-466.

Kourtev PS, Huang WZ, Ehrenfeld JG (1999). Differences in earthworm densities and nitrogen dynamics in soils under exotic and native plant species. Biological Invasions, $1(2-3), 237-245$.

Lee MR, Bernhardt ES, Bodegom PM, Cornelissen JHC, Kattge J, Laughlin DC, Niinemets U, Penuelas J, Reich PB, Yguel B, Wright JP (2016). Invasive species' leaf traits and dissimilarity from natives shape their impact on nitrogen cycling: A meta-analysis. New Phytologist, 213, 128-139.

Lei YB, Xiao HF, Feng YL (2010). Impacts of alien plant invasions on biodiversity and evolutionary responses of native species. Biodiversity Science, 18, 622-630. [类延宝, 肖海 峰, 冯玉龙 (2010). 外来植物入侵对生物多样性的影响 及本地生物的进化响应. 生物多样性, 18, 622-630.]

Li B, Ma KP (2010). Biological invasions: Opportunities and challenges facing Chinese ecologists in the era of translational ecology. Biodiversity Science, 18, 529-532. [李博, 马克平 (2010). 生物入侵: 中国学者面临的转化生态
学机遇与挑战. 生物多样性, 18, 529-532.]

Li WH, Zhang CB, Gao GJ, Zan QJ, Yang ZY (2007). Relationship between Mikania micrantha invasion and soil microbial biomass, respiration and functional diversity. Plant and Soil, 296, 197-207.

Liao CZ, Peng R, Luo Y, Zhou XH, Wu XW, Fang CM, Chen JK, Li B (2008). Altered ecosystem carbon and nitrogen cycles by plant invasion: A meta-analysis. New Phytologist, 177, 706-714.

Liao JD, Boutton TW, Jastrow JD (2006). Organic matter turnover in soil physical fractions following woody plant invasion of grassland: Evidence from natural ${ }^{13} \mathrm{C}$ and ${ }^{15} \mathrm{~N}$. Soil Biology and Biochemistry, 38, 3197-3210.

Lu X, Bottomley PJ, Myrold DD (2015). Contributions of ammonia-oxidizing archaea and bacteria to nitrification in Oregon forest soils. Soil Biology and Biochemistry, 85, 54-62.

Marchante E, Kjøller A, Struwe S, Freitas H (2008). Short- and long-term impacts of Acacia longifolia invasion on the belowground processes of a Mediterranean coastal dune ecosystem. Applied Soil Ecology, 40, 210-217.

Martinelli LA, Piccolo MC, Townsend AR, Vitousek PM, Cuevas E, Mcdowell W, Robertson GP, Santos OC, Treseder K (1999). Nitrogen stable isotopic composition of leaves and soil: Tropical versus temperate forests. Biogeochemistry, 46(1-3), 45-65.

McLeod ML, Cleveland CC, Lekberg Y, Maron JL, Philippot L, Bru D, Callaway RM (2016). Exotic invasive plants increase productivity, abundance of ammonia-oxidizing bacteria and nitrogen availability in intermountain grasslands. Journal of Ecology, 104, 994-1002.

Milbau A, Nijs I, Peer LV, Reheul D, Cauwer BD (2003). Disentangling invasiveness and invasibility during invasion in synthesized grassland communities. New Phytologist, 159, 657-667.

Niu HB, Liu WX, Wan FH, Liu B (2007). An invasive aster (Ageratina adenophora) invades and dominates forest understories in China: Altered soil microbial communities facilitate the invader and inhibit natives. Plant and Soil, 294, 73-85.

Peña EDL, Clercq ND, Bonte D, Roiloa S, RodríguezEcheverría S, Freitas H (2010). Plant-soil feedback as a mechanism of invasion by Carpobrotus edulis. Biological Invasions, 12, 3637-3648.

Perakis SS, Hibbs DE (2014). $\mathrm{N}_{2}$-fixing red alder indirectly accelerates ecosystem nitrogen cycling. Ecosystems, 15, $1182-1193$. 
Pimentel D, Zuniga R, Morrison D (2005). Update on the environmental and economic costs associated with alien- invasive species in the United States. Ecological Economics, 52, 273-288.

Polyakova O, Billor N (2008). Impact of deciduous tree species on litterfall quality, decomposition rates and nutrient circulation in pine stands. Forest Ecology and Management, 253, 11-18.

Reich PB, Oleksyn J (2004). Global patterns of plant leaf N and $\mathrm{P}$ in relation to temperature and latitude. Proceedings of the National Academy of Sciences of the United States of America, 101, 11001-11006.

Rossiter-Rachor NA, Setterfield SA, Douglas MM, Hutley LB, Cook GD, Schmidt S (2009). Invasive Andropogon gayanus (gamba grass) is an ecosystem transformer of nitrogen relations in Australian savanna. Ecological Applications, 19, 1546-1560.

Sardans J, Bartrons M, Margalef O, Gargallo-Garriga A, Janssens IA, Ciais P, Obersteiner M, Sigurdsson BD, Chen HH, Penuelas J (2016). Plant invasion is associated with higher plant-soil nutrient concentrations in nutrient-poor environments. Global Change Biology, 23, 1282-1291.

Schittko C, Runge C, Strupp M, Wolff S, Wurst S (2016). No evidence that plant-soil feedback effects of native and invasive plant species under glasshouse conditions are reflected in the field. Journal of Ecology, 104, 1243-1249.

Shannon-Firestone S, Reynolds HL, Phillips RP, Flory SL, Yanarell A (2015). The role of ammonium oxidizing communities in mediating effects of an invasive plant on soil nitrification. Soil Biology and Biochemistry, 90, 266-274.

Solly EF, Schöning I, Boch S, Kandeler E, Marhan S, Michalzik B, Müller J, Zscheischler J, Trumbore SE, Schrumpf M (2014). Factors controlling decomposition rates of fine root litter in temperate forests and grasslands. Plant and Soil, 382, 203-218.

Souza-Alonso P, Novoa A, González L (2014). Soil biochemical alterations and microbial community responses under Acacia dealbata Link invasion. Soil Biology and Biochemistry, 79, 100-108.

Strickland MS, Devore JL, Maerz JC, Bradford MA (2010). Grass invasion of a hardwood forest is associated with declines in belowground carbon pools. Global Change Biology, 16, 1338-1350.

Sun X, Gao C, Guo LD (2013). Changes in soil microbial community and enzyme activity along an exotic plant Eupatorium adenophorum invasion in a Chinese secondary forest. Science Bulletin, 58, 4101-4108.

Tang YQ, Yu GR, Zhang XY, Wang QF, Ge JP, Liu S (2017). Changes in nitrogen-cycling microbial communities with depth in temperate and subtropical forest soils. Applied Soil Ecology, 124, 218-228.

Theoharides KA, Dukes JS (2007). Plant invasion across space and time: Factors affecting nonindigenous species success during four stages of invasion. New Phytologist, 176, 256-273.

van Kleunen M, Weber E, Fischer M (2010). A meta-analysis of trait differences between invasive and non-invasive plant species. Ecology Letters, 13, 235-245.

Vilà M, Espinar JL, Hejda M, Hulme PE, Jarošík V, Maron JL, Pergl J, Schaffner U, Sun Y, Pyšek P (2011). Ecological impacts of invasive alien plants: A meta-analysis of their effects on species, communities and ecosystems. Ecology Letters, 14, 702-708.

Vitousek PM, Cassman K, Cleveland C, Crews T, Field CB, Grimm NB, Howarth RW, Marino R, Martinelli L, Rastetter EB, Sprent JI (2002). Towards an ecological understanding of biological nitrogen fixation. Biogeochemistry, 57, 1-45.

Vitousek PM, Matson PA (1984). Mechanisms of nitrogen retention in forest ecosystems: A field experiment. Science, 225, 51-52.

Wang CH, Wan SQ, Xing XR, Zhang L, Han XG (2006). Temperature and soil moisture interactively affected soil net $\mathrm{N}$ mineralization in temperate grassland in Northern China. Soil Biology and Biochemistry, 38, 1101-1110.

Wei H, Xu JL, Quan GM, Zhang JE, Qin Z (2017). Effects of Praxelis clematidea invasion on soil nitrogen fractions and transformation rates in a tropical savanna. Environmental Science and Pollution Research, 24, 1-10.

Xiao HF, Feng YL, Schaefer DA, Xiao DY (2014). Soil fungi rather than bacteria were modified by invasive plants, and that benefited invasive plant growth. Plant and Soil, 378, 253-264.

Xu CW, Yang MZ, Chen YJ, Chen LM, Zhang DZ, Mei L, Shi YT, Zhang HB (2012). Changes in non-symbiotic nitrogen-fixing bacteria inhabiting rhizosphere soils of an invasive plant Ageratina adenophora. Applied Soil Ecology, 54, 32-38.

Yan XL, Shou HY, Ma JS (2012). The problem and status of the alien invasive plants in China. Plant Diversity and Resources, 34, 287-313. [闵小玲, 寿海洋, 马金双 (2012). 中国外来入侵植物研究现状及存在的问题. 植物分类 与资源学报, 34, 287-313.] 
Yang O, Norton JM, Stark JM (2017). Ammonium availability and temperature control contributions of ammonia oxidizing bacteria and archaea to nitrification in an agricultural soil. Soil Biology and Biochemistry, 113, 161-172.

Yé L, Lata JC, Masse D, Nacro HB, Kissou R, Diallo NH, Barot S (2017). Contrasted effects of annual and perennial grasses on soil chemical and biological characteristics of a grazed Sudanian savanna. Applied Soil Ecology, 113, $155-165$.

Yu XJ, Yu D, Lu ZJ, Ma KP (2005). A new mechanism of invader success: Exotic plant inhibits natural vegetation restoration by changing soil microbe community. Chinese Science Bulletin, 50, 896-903. [于兴军, 于丹, 卢志军, 马克平 (2005). 一个可能的植物入侵机制: 入侵种通
过改变入侵地土壤微生物群落影响本地种的生长. 科 学通报, 50, 896-903.]

Zhang YH, Ding WX, Luo JF, Donnison A (2010). Changes in soil organic carbon dynamics in an eastern Chinese coastal wetland following invasion by a $\mathrm{C}_{4}$ plant Spartina alterniflora. Soil Biology and Biochemistry, 42, 1712-1720.

Zheng YL, Burns JH, Liao ZY, Li YP, Yang J, Chen YJ, Zhang JL, Zheng YG (2018). Species composition, functional and phylogenetic distances correlate with success of invasive Chromolaena odorata in an experimental test. Ecology Letters, 21, 1211-1220.

责任编委: 何伟明 责任编辑: 李 敏 